\title{
Energy and macronutrient intakes across the lifecourse from current national dietary surveys of European populations
}

\author{
H.L. Rippin ${ }^{1}$, J. Hutchinson ${ }^{1}$, J. Jewell ${ }^{2}$, J.J. Breda ${ }^{2}$ and J.E. Cade ${ }^{1}$ \\ ${ }^{1}$ Nutritional Epidemiology Group (NEG), School of Food Science and Nutrition, University of Leeds, Leeds, LS2 9JT \\ and ${ }^{2}$ Division of Noncommunicable Diseases and Promoting Health through the Life-Course, World Health \\ Organization Regional Office for Europe, UN City, Marmorvej 51, 21000 Copenhagen, Denmark
}

The World Health Organisation (WHO) encourages countries to undertake national diet surveys (NDS) to inform policies addressing nutrition-related health problems, such as obesity, which remains a pressing health issue in adults and children across Europe ${ }^{(1)}$. An up-to-date review of adult and child energy and selected macronutrient intakes from NDS in the 53 countries of the WHO European region was undertaken and these were assessed against WHO Recommended Nutrient Intakes (RNIs) ${ }^{(2,3)}$, to highlight patterns and areas of concern.

Information on NDS of whole diets, conducted post-1990, with sample size $>200$ in the WHO European Region was gathered primarily by internet searches and contacting survey authors and nutrition experts. A systematic literature database search returned few relevant results. Survey characteristics were tabled from all relevant NDS, and most recent energy and nutrient intakes extracted from the latest NDS conducted for each country after 2000. Weighted mean intakes for adults and children were calculated by country, European area and overall.

111 NDS collected post-1990 were found across 34 countries; 43 NDS surveyed adults, 22 surveyed children and 46 included both. Over a third $(n=19)$ of the 53 countries in the WHO European Region, mainly Central \& Eastern European countries (CEEC), had no identifiable NDS. Energy and nutrient intakes were extracted from 21 NDS across 21 countries for adults and 21 NDS across 18 countries for children, though not all countries reported all nutrients. Differences in age grouping, dietary assessment methodology, under-reporting and nutrient composition databases also hindered inter-country comparisons. Men were more likely than women to have elevated energy intakes; in two thirds countries $(n=14)$ adult men of all ages exceeded UK energy Reference Intakes ${ }^{(4)}$, compared to $10 \%(\mathrm{n}=2)$ for women. Macronutrient RNI attainment was poor across the lifecourse; no adults and few children met the carbohydrate, added sugar or fat RNIs and few countries met the fibre or saturated fat RNIs in any age (fig. 1).

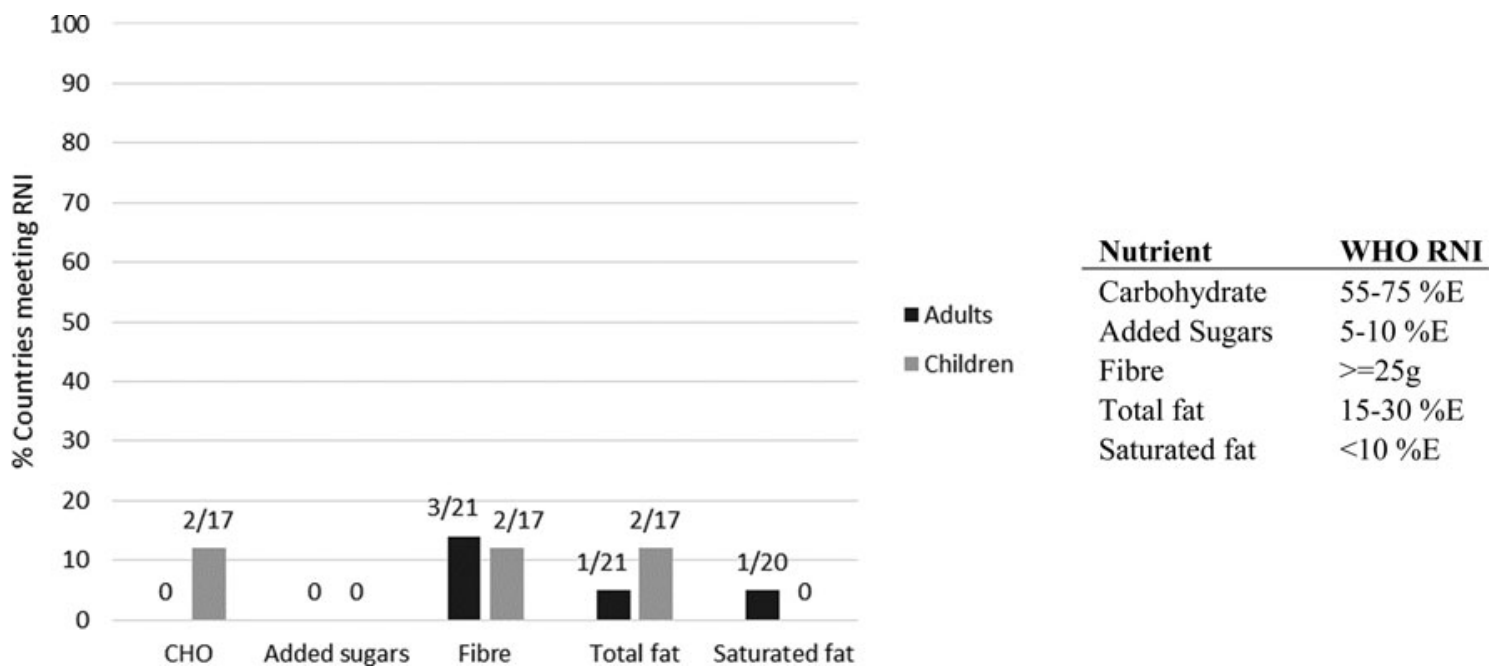

Fig. 1. \% Countries meeting WHO macronutrient RNIs, where reported, in at least one age/gender group.

Less than two thirds of WHO European Region countries have NDS, and less than half provide adult and/or child energy and nutrient intakes. The main gaps lie in CEEC, where nutrition policies may lack an evidence base and dietary inadequacies go unidentified. WHO RNI attainment was universally poor, but methodological and other inconsistencies hinder inter-country comparisons. All countries should be encouraged to conduct and report NDS in a standardised format to aid obesity-prevention policy formation.

1. World Health Organisation (2014) European food and nutrition action plan 2015-020. Copenhagen: WHO.

2. World Health Organisation (2003) Diet, nutrition and the prevention of chronic diseases. Joint WHO/FAO Expert Consultation. WHO Technical Report Series no.916. Geneva: WHO.

3. World Health Organisation (2015) Guideline: sugars intake for adults and children. Geneva: WHO.

4. British Nutrition Foundation (2016) Nutrition requirements. London: BNF. https://www.nutrition.org.uk/attachments/article/234/ Nutrition20Requirements_Revised20Oct\%202016.pdf (accessed March 2018). 